\title{
MONITORAMENTO CONTÍNUO DO POTENCIAL REDOX E DE VARIÁVEIS COMPLEMENTARES EM AMBIENTE HIPERSAZONAL NO PANTANAL NORTE ${ }^{(1)}$
}

\author{
Indira Ashant Martins Messias ${ }^{(2)}$, Eduardo Guimarães Couto ${ }^{(3)}$, Ricardo Silva Santos \\ Amorim $^{(5)}$, Mark Stephen Johnson ${ }^{(6)}$ \& Osvaldo Borges Pinto Junior ${ }^{(4)}$
}

\section{RESUMO}

A análise conjunta do potencial redox com outras variáveis pode ser útil para o entendimento da dinâmica dos solos hidromórficos, ao longo de todo o ciclo hidrológico a que o Pantanal está submetido. Neste trabalho, foram estudados o potencial redox e algumas variáveis do solo $\left(\mathrm{CO}_{2}, \mathrm{O}_{2}\right.$, temperatura, umidade e potencial matricial) pelo monitoramento contínuo e ininterrupto. Para isso, foram realizadas determinações nas profundidades de 10 e $30 \mathrm{~cm}$ num Planossolo Háplico alítico gleissólico, na fitofisionomia Cerrado sensu stricto. $O$ trabalho foi realizado de fevereiro de 2010 a julho de 2011 na RPPN (Reserva Particular do Patrimônio Natural/Sistema Nacional de Unidades de Conservação - SNUC), Estância Ecológica SESC (Serviço Social do Comércio) Pantanal, município de Barão de Melgaço, nordeste do Pantanal de Mato Grosso. Os valores do potencial redox variaram de $636 \mathrm{mV}$ (enchente) a -341 $\mathrm{mV}$ (cheia), caracterizando ambiente anaeróbico na estação da cheia e ambiente oxidado nas demais estações do ciclo hidrológico, o que indica que o potencial redox é ferramenta versátil para compreender as reações de oxirredução nos solos hidromórficos do Pantanal mato-grossense, pois os valores diminuíram quando o teor de umidade era elevado. Além disto, o estudo evidenciou também que o monitoramento contínuo de variáveis complementares pode ser importante por permitir análise mais aprofundada das condições de hipersazonalidade, em que esses solos estão submetidos. Neste contexto, o potencial matricial e a umidade foram as variáveis de maior importância para explicar a variação do conjunto de dados obtidos ao longo do ciclo hidrológico, o que indica

(1) Artigo extraído da Dissertação de Mestrado do primeiro autor. Recebido para publicação em 26 de setembro de 2011 e aprovado em 14 de abril de 2013.

(2) Pós-graduação em Agricultura Tropical; Universidade Federal de Mato Grosso - UFMT. Av. Fernando Corrêa da Costa, s/n. CEP 78069-0000 Cuiabá (MT). E-mail: indiramessias@gmail.com

(3) Professor Associado, Departamento de Solos e Engenharia Rural, UFMT. E-mail: couto@ufmt.br

(4) Pós-doutor na Pós-graduação em Física Ambiental, Instituto de Física, UFMT. E-mail: osvaldo.borges@gmail.com

(5) Professor Adjunto, Departamento de Solos e Engenharia Rural, UFMT. E-mail: rsamorim@ufmt.br

(6) Professor do Instituto de Recursos, Meio Ambiente e Sustentabilidade, Departamento de Terra e Ciências Oceânicas, Universidade de British Columbia, 2202. Main Mall, USA. E-mail: mark.johnson@ubc.ca 


\author{
que esses parâmetros físicos são determinantes nos processos biológicos desse \\ solo tropical com hipersazonalidade hídrica.
}

Termos de indexação: sazonalidade, ciclo hidrológico, Eh.

\author{
SUMMARY: MONITORING THE SOIL REDOX POTENTIAL AND ITS \\ INTERACTIONS WITH ASSOCIATED VARIABLES IN THE \\ NORTHERN PANTANAL
}

\begin{abstract}
The analysis of redox potential and other associated soil variables may be useful for understanding the dynamics of hydromorphic soils throughout the water cycle of the Pantanal. This study investigated the redox potential and soil variables $\left(\mathrm{CO}_{2}, \mathrm{O}_{2}\right.$, temperature, moisture and matric potential) by continuous and uninterrupted monitoring. Measurements were made at 10 and $30 \mathrm{~cm}$ depth in a Haplic alitic gleisolic Planossol soil with Cerrado sensu stricto vegetation, in the municipality of Barão do Melgaço in the northeastern Pantanal. Redox potential values ranged from $636 \mathrm{mV}$ (rising water period) to $-341 \mathrm{mV}$ (flooded period), characterizing an anaerobic environment when flooded and an oxidized environment in the other seasons of the hydrological cycle. This confirmed the redox potential as a versatile indicator to understand the oxidative-reductive reactions in the hydromorphic soils of the Pantanal wetlands, since the redox values decreased when soil moisture was high. The study also showed that continuous and uninterrupted monitoring of additional variables can be important for further analyses of the hyperseasonal nature of these soils. Soil matrix potential and soil moisture were the variables that explained most variation in the data of the hydrological cycle, indicating that these physical parameters are crucial for biological processes in this tropical soil with hydric hyperseasonality.
\end{abstract}

Index terms: seasonality, hydrologic cycle, Eh.

\section{INTRODUÇÃO}

O Pantanal mato-grossense apresenta alterações sazonais ao longo do ano, que se caracterizam por uma estação seca e uma chuvosa. O ciclo de inundação é monomodal (Junk et al., 1989) e, de ano para ano, as cheias apresentam diferenças de amplitude, com variação no tempo e espaço (Girard et al., 2010).

Em razão da inundação do monomodal, várias regiões desse bioma mudam de habitat aquático para terrestre e vice-versa, ocasionando cheias que atingem $80 \%$ do Pantanal (Lobato, 2000).

Os dados sobre potencial redox podem ser ferramentas versáteis para compreender as reações de oxirredução nos solos (Vepraskas \& Faulkner, 2001), especialmente aqueles submetidos à sazonalidade de cheia (fase anaeróbica) e seca (fase aeróbica).

Aliada às determinações de potencial redox, a análise da respiração do solo e da difusão do oxigênio $\left(\mathrm{O}_{2}\right)$ e dióxido de carbono $\left(\mathrm{CO}_{2}\right)$ é de extrema importância, pois as mudanças do potencial redox são o resultado de efeitos combinados de processos químicos, físicos e biológicos no solo (Vepraskas \& Faulkner, 2001). Considerando a importância de se estudar o potencial redox em áreas úmidas com outras variáveis para compreender a dinâmica de solos hidromórficos, os objetivos deste trabalho foram caracterizar e monitorar o potencial redox e outras variáveis complementares $\left(\mathrm{CO}_{2}, \mathrm{O}_{2}\right.$, temperatura, umidade, potencial matricial) no solo de uma cordilheira, na fitofisionomia Cerrado sensu stricto, localizada na Reserva Particular do Patrimônio Natural - SESC Pantanal.

\section{MATERIAL E MÉTODOS}

\section{Área de estudo}

O trabalho foi realizado de fevereiro de 2010 a julho de 2011 na RPPN (Reserva Particular do Patrimônio Natural/Sistema Nacional de Unidades de Conservação - SNUC), Estância Ecológica SESC (Serviço Social do Comércio), Pantanal, município de Barão de Melgaço, nordeste do Pantanal de Mato Grosso (Figura 1), localizada entre as coordenadas geográficas $16^{\circ} 30^{\prime}$ a $16^{\circ} 44^{\prime} \mathrm{S}$ e $56^{\circ} 20^{\prime}$ a $56^{\circ} 30^{\prime} \mathrm{W}$, ocupando uma área de aproximadamente $1.076 \mathrm{~km}^{2}$. Em 2002, a RPPN foi reconhecida como um site Ramsar - Convenção sobre Zona Úmidas, Ramsar, Irã, de 1971 - e é considerada uma área prioritária para conservação da biodiversidade (Cordeiro, 2004). Foi também incluída na lista de Áreas Úmidas da Convenção Ramsar, de importância internacional (Junk \& Cunha, 2005).

O clima da região é tipo Aw, segundo a classificação de Köppen, apresentando sazonalidade característica, 


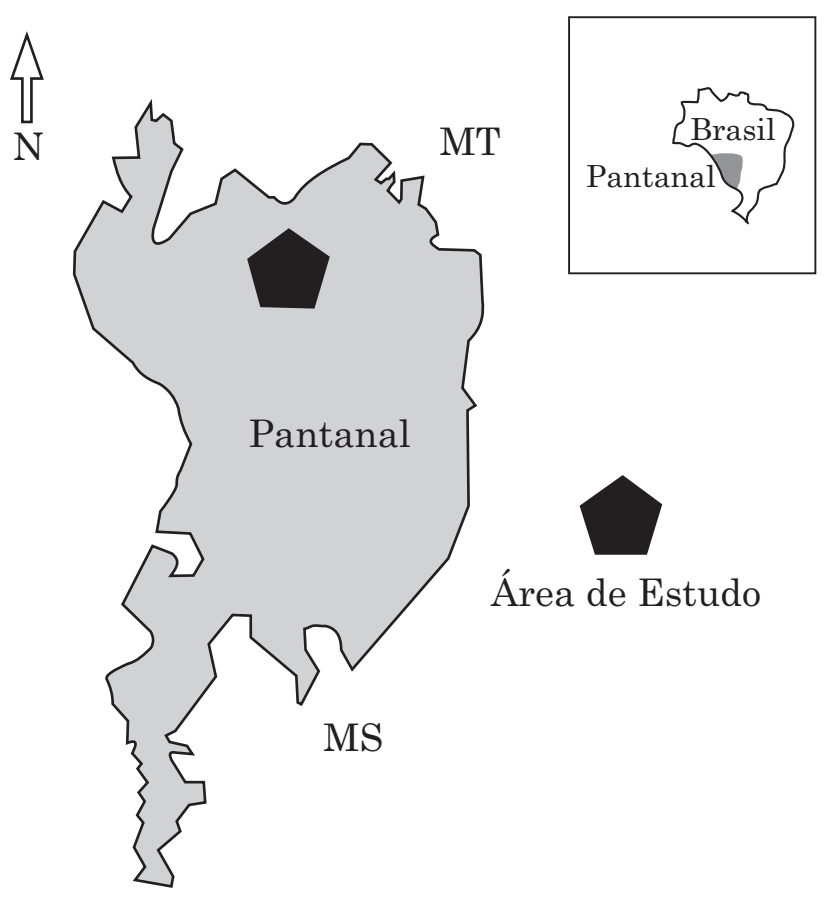

Figura 1. Localização da área de estudo.

com uma estação chuvosa (outubro-abril) e outra seca (maio-setembro) (Cunha \& Junk, 2004). A temperatura média do ar oscila entre 22 a $32{ }^{\circ} \mathrm{C}$ ao longo do ano e a precipitação pluvial média anual varia entre 1.100 e $1.200 \mathrm{~mm}$, com o total médio do mês mais seco inferior a $40 \mathrm{~mm}$ (Hasenack et al., 2003).

O solo foi classificado como Planossolo Háplico alítico gleissólico e a fitofisionomia se presenta como Cerrado sensu stricto. Os solos dessa classe apresentam mudança textural abrupta com acentuada diferença de textura do A para o horizonte B, imediatamente subjacente, permeabilidade lenta ou muito lenta, profundidade de $150 \mathrm{~cm}$ e textura arenosa.

\section{Medidas de Campo}

O ciclo hidrológico foi dividido em quatro estações: a estação da "cheia" corresponde aos meses de fevereiro, março e abril; a estação da "vazante", aos meses de maio, junho e julho; a estação da "estiagem", aos meses de agosto, setembro e outubro; e, por fim, a estação da "enchente", aos meses de novembro, dezembro e janeiro. Os períodos foram divididos em manhã, tarde e noite: o período da manhã é das 7 às 13h30; o da tarde, das 14 às 18h30; e o da noite, das 19 às $6 \mathrm{~h} 30$.

Foram instalados sensores horizontalmente no perfil do solo na borda de uma "cordilheira", nas profundidades de 10 e $30 \mathrm{~cm}$ para o monitoramento contínuo da concentração de $\mathrm{CO}_{2}$ (ppm), do conteúdo de $\mathrm{O}_{2}(\%)$, da temperatura $\left({ }^{\circ} \mathrm{C}\right)$, da umidade do solo $\left(\mathrm{cm}^{3} \mathrm{~cm}^{-3}\right)$, do potencial matricial $(\mathrm{kPa})$ e do potencial redox $(\mathrm{mV})$, por meio de um datalogger modelo CR1000
(Campbell Scientific, Logan, Utah, USA). O datalogger foi conectado a uma bateria de $12 \mathrm{~V}$ com uma placa solar ( $45 \mathrm{~W})$, o que garantia energia suficiente para o monitoramento contínuo e ininterrupto.

A concentração de $\mathrm{CO}_{2}$ no solo foi determinada in situ por meio de um analisador de gás por infravermelho (Jassal et al., 2004; Johnson et al., 2010). Durante os primeiros 5 min de cada meia hora, os sensores eram ligados em razão do alto consumo de energia (4W cada) (Vaisala Oyj, 2008). Nesse intervalo, os primeiros 3 min em que os sensores ficavam ligados correspondiam ao seu período de aquecimento; os subsequentes 2 min restantes correspondiam às leituras feitas num intervalo de $30 \mathrm{~s}$, resultando em registros de meia em meia hora.

O potencial redox foi determinado pelo sensor ColeParmer, que utiliza uma área superficial de platina ( 0,2 pol em torno do eletrodo de vidro), permitindo a sensibilidade do sensor a atividades de elétrons do fluido. $\mathrm{O}$ sensor de potencial redox de $30 \mathrm{~cm}$ foi instalado em fevereiro de 2010 e o de potencial redox de $10 \mathrm{~cm}$, em setembro do mesmo ano. Os dados de potencial redox foram corrigidos pela equação de regressão, equação 1 , proposta por Vepraskas \& Faulkner (2001).

$$
\text { Fator de ajuste }=-0,6743 \times(T)+213,76
$$

em que T é a temperatura $\left({ }^{\circ} \mathrm{C}\right)$ na profundidade do sensor redox.

Potencial redox $(\mathrm{Eh}, \mathrm{mV})=$ fator de ajuste + medida do sensor redox

As determinações de pressão atmosférica e temperatura do solo foram usadas para corrigir as leituras de concentração de $\mathrm{CO}_{2}$ pela dependência desses valores à lei dos gases perfeitos (Vaisala Oyj, 2008).

A umidade do solo foi estimada por sensores com base no domínio da capacitância/frequência (Decagon Instruments, model EC-5). O potencial matricial do solo (potencial matricial) foi analisado usando um sensor desse mesmo fabricante (Decagon Instruments, model MPS-1); todas as variáveis foram definidas em intervalos de $30 \mathrm{~min}$.

$\mathrm{O} \mathrm{O}_{2}$ do solo foi estabelecido por meio de um sensor de células galvânicas no estado sólido (Kallestad et al., 2008). O sensor de oxigênio inclui um termostato para determinar a temperatura do ambiente. Durante os meses que correspondiam às estações da estiagem e enchente, o sensor de oxigênio de $30 \mathrm{~cm}$ apresentou problemas técnicos comprometendo a produção de informações nesse período. Além disso, o pH da solução do solo extraído de um coletor próximo aos sensores foi medido mesalmente no ano de 2010 (Beirigo, 2011).

\section{Análises estatísticas}

Os valores de concentração de $\mathrm{CO}_{2}$ foram estimados por interpolação linear, quando a distância dos valores 
de antes e depois (que não saíssem do intervalo) não fossem maiores que $48 \mathrm{~h}$. Esse procedimento foi automatizado, utilizando o software de séries temporais Aquarius, evitando a subjetividade das estimativas.

\section{RESULTADOS E DISCUSSÃO}

\section{Monitoramento e caracterização das variáveis do solo e micrometereológicas}

A variação do potencial redox para o solo estudado está na figura 2 , em que foram plotados os dados médios determinados pelo sensor. Entre abril de 2010 e fevereiro de 2011, o solo esteve insaturado, ou seja, em estado de oxidação; o intervalo de valores nesse período foi de $400 \mathrm{mV}$. No período de saturação, março de 2010 e julho de 2011, a variação de valores foi superior a $1000 \mathrm{mV}$.

O tipo de variabilidade é esperado para solos submetidos a uma sazonalidade de seca e cheia, caso do solo estudado no Pantanal de Mato Grosso (Figura 2). Quando o potencial redox ficou maior que $300 \mathrm{mV}$ na profundidade de $10 \mathrm{~cm}$, o solo estava insaturado e o $\mathrm{O}_{2}$ controlava o sistema. $\mathrm{Na}$ ausência de $\mathrm{O}_{2}$, os microrganismos reduzem uma variedade de aceptores de elétrons considerados alternativos, que, em ordem decrescente de termodinâmica são: $\mathrm{NO}_{3}{ }^{-}$ (denitrificação), Fe (III) (redução do ferro), Mn (III, IV) (redução do manganês) e $\mathrm{SO}_{4}^{-}$(redução do sulfato) (Keller et al., 2009).

A linha que evidencia a fase de mudança de $\mathrm{Fe}(\mathrm{OH})_{3}$ para $\mathrm{Fe}^{2+}($ Vepraskas \& Faulkner, 2001) foi adicionada à figura 2 pela linha horizontal no valor de Eh de $300 \mathrm{mV}$, de acordo com o pH médio da solução do solo do local de estudo, que foi aproximadamente 6,0 . Durante o mês de março, o $\mathrm{Fe}^{2+}$ estava na solução

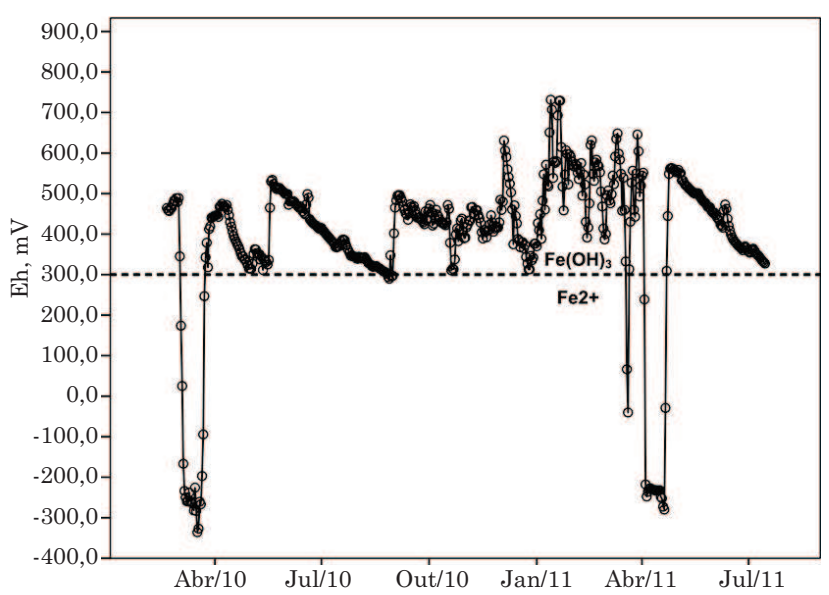

Figura 2. Variação (média diária) do potencial redox, na profundidade de $10 \mathrm{~cm}$, num solo classificado como Planossolo Háplico alítico gleissólico, na fitofisionomia Cerrado sensu stricto. do solo, ou seja, se o $\mathrm{Fe}^{2+}$ estava presente, pode-se assumir que a maior parte do $\mathrm{O}_{2}$ dissolvido foi reduzido para $\mathrm{H}_{2} \mathrm{O}$; a maior parte do $\mathrm{NO}_{3}^{-}$presente foi desnitrificada, bem como a dos óxidos de Mn, reduzida para $\mathrm{Mn}^{2+}$.

A redução do $\mathrm{Mn}$ (IV) ocorre a valores de Eh maiores que do $\mathrm{Fe}^{3+}$, geralmente entre 200 e $300 \mathrm{mV}$, segundo Sousa et al. (2009), podendo ser mobilizado até mesmo em ambiente ligeiramente reduzido; consequentemente, ocorre acúmulo de $\mathrm{Mn}^{2+}$ em $\mathrm{Eh}$ mais elevado do que o $\mathrm{Fe}^{2+}$, sugerindo que a redução do $\mathrm{Mn}^{4+}$ preceda o $\mathrm{Fe}^{3+}$ (Neaman et al., 2004).

Com a redução dos compostos do solo pela inundação, o pH de solos ácidos aumenta, assim como os teores de $\mathrm{Fe}^{2+} \mathrm{e} \mathrm{Mn}^{2+}$ em solução (Lima et al., 2005). Quando o $\mathrm{pH}$ dos solos atinge valores próximos a 6,57,0 , estabelecem-se condições para a reoxidação do $\mathrm{Fe}^{2+}$ por reações químicas e microbiológicas (Liesack et al., 2000). Esse ambiente químico reduzido é de grande importância ecológica, pois a mudança no estado de oxirredução modifica a biodisponibilidade e mobilidade dos diferentes elementos presentes no solo.

O potencial redox diminuiu na cheia (meados de fevereiro), ou seja, em que o teor de umidade elevado diminuiu a taxa e quantidade de difusão de $\mathrm{O}_{2}$ no solo e aumentou nas outras estações do ciclo hidrológico. Nas estações secas do Pantanal, o solo encontra-se com baixo teor de umidade havendo a predominância de valores positivos, que são típicos de solos aeróbicos (Ponnomperumo, 1972), demonstrando predominarem condições não limitantes ao crescimento de plantas (Volk, 1939).

Os valores médios do potencial redox nas profundidades de 10 e $30 \mathrm{~cm}$ (Figura 3) foram estatisticamente diferentes nas estações de cheia, vazante e estiagem. Já se tratando dos períodos manhã, tarde e noite, não houve diferença estatística em nenhuma das estações estudadas em ambas as profundidades. Observou-se que as médias na profundidade de $10 \mathrm{~cm}$ nas estações de cheia, vazante e estiagem foram menores e diferiram significativamente das médias da profundidade de $30 \mathrm{~cm}$. Para o período da enchente, não houve diferença entre as profundidades.

Os metais presentes em $\mathrm{Eh}<150 \mathrm{mV}$ estão unidos a espécies de sulfatos, formando $\mathrm{FeS}_{2}, \mathrm{MnSO}_{4}$, entre outros, enquanto em valores $>150 \mathrm{mV}$ (todas as estações em ambas as profundidades), a maioria dos metais presentes são solúveis e unidos às espécies oxidadas $\mathrm{Fe}_{3} \mathrm{O}_{4}, \mathrm{Mn}_{2} \mathrm{O}_{4}$ (Clark et al., 1998; Vepraskas \& Faulkner, 2001).

O potencial redox foi geralmente maior na profundidade de $30 \mathrm{~cm}$ do que perto da superfície do solo $(10 \mathrm{~cm})$, o que deve estar relacionado ao maior teor de matéria orgânica na superfície dos solos (Lima et al., 2005). A matéria orgânica do solo influencia a dinâmica do $\mathrm{Fe}$ e do $\mathrm{Mn}$, tanto por seu efeito inibidor do processo de cristalinidade dos óxidos (Jansen et al., 2004) quanto por sua atuação como fonte de energia 


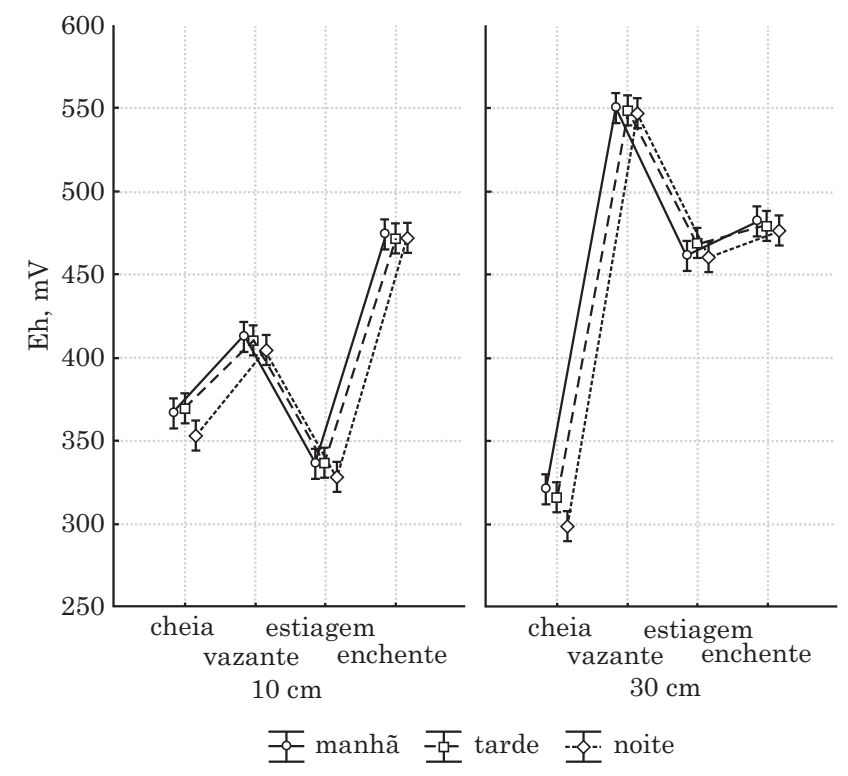

Figura 3. Médias, por ciclo hidrológico, do potencial redox, nas profundidades de 10 e $30 \mathrm{~cm}$, num solo classificado como Planossolo Háplico alítico gleissólico, na fitofisionomia Cerrado sensu stricto. As barras expressam o intervalo de confiança a $95 \%$.

para os microrganismos responsáveis pela redução microbiana dos compostos oxidados. Elevados teores de matéria orgânica favorecem a formação de complexos de $\mathrm{Fe}^{3+}$ solúveis e suportam a formação da ferrihidrita (Rancourt et al., 2005).

Os valores de $\mathrm{CO}_{2}$ no solo na profundidade de 10 $\mathrm{cm}$ foram mais altos na cheia e enchente do que na vazante e estiagem (Figura 4). Na profundidade de $30 \mathrm{~cm}$, os maiores valores também foram encontrados nas estações de cheia e enchente, enquanto os menores, nas estações vazante e estiagem. A produção de $\mathrm{CO}_{2}$ no solo aumentou nas chuvas no período da enchente, o que explica a diferença entre a estiagem e enchente. Os valores médios diferiram entre si ao longo do ciclo hidrológico como também entre as profundidades. Dentro de cada estação, os valores foram menores na profundidade de $10 \mathrm{~cm}$ do que em $30 \mathrm{~cm}$.

Os solos secos podem ser associados a baixas concentrações de $\mathrm{CO}_{2}$, visto que possuem alta difusividade, permitindo rápido efluxo de $\mathrm{CO}_{2}$ do solo para a atmosfera (Risk et al., 2008). Tal dinâmica foi observada na profundidade de $10 \mathrm{~cm}$ na estação da estiagem, com altas concentrações de $\mathrm{O}_{2}$ (Figura 5) e baixas de $\mathrm{CO}_{2}$ (Figura 4). As altas concentrações de $\mathrm{CO}_{2}$ encontradas na cheia nas profundidades de $10 \mathrm{e}$ $30 \mathrm{~cm}$ indicaram condições de difusão limitada no solo (De Jonge \& Mittelmeijer-Hazeleger, 1996).

$\mathrm{O}$ aumento de concentração de $\mathrm{CO}_{2}$ nos primeiros $10 \mathrm{~cm}$ continua da enchente para a cheia, podendo ainda continuar crescendo durante a cheia, desde que o solo não esteja saturado. Em condições anaeróbicas

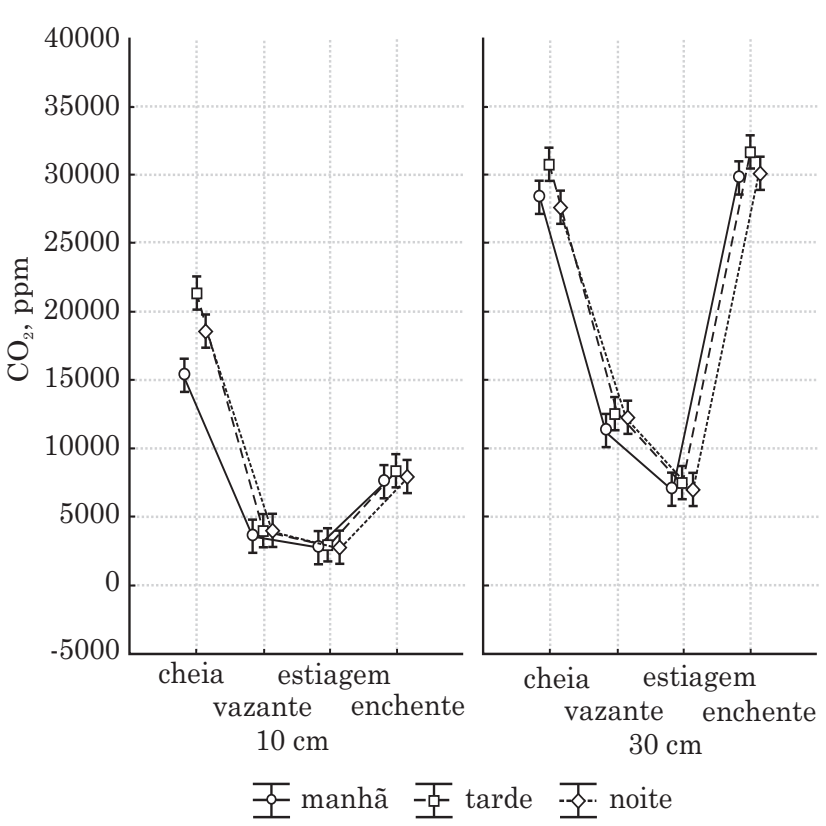

Figura 4. Médias, por ciclo hidrológico, do $\mathrm{CO}_{2}$, nas profundidades de 10 e $30 \mathrm{~cm}$, num solo classificado como Planossolo Háplico alítico gleissólico, na fitofisionomia Cerrado sensu stricto. As barras expressam o intervalo de confiança a $95 \%$.

não existe produção de $\mathrm{CO}_{2}$, mas sim de metano (Luo $\&$ Zhou, 2006). Além disso, Bowden et al. (1993) e Xu et al. (2004) sugeriram também que o efluxo de $\mathrm{CO}_{2}$ pode ser limitado pela umidade em níveis muito altos. Assim, a concentração de $\mathrm{CO}_{2}$ na profundidade de $10 \mathrm{~cm}$ pode resultar da produção de $\mathrm{CO}_{2}$ nesse local, como também do transporte lento de $\mathrm{CO}_{2}$, proveniente das camadas inferiores, ou uma combinação desses fatores.

Os valores médios de $\mathrm{O}_{2}$ do solo (Figura 5) foram diferentes entre as estações e só houve diferença significativa entre os períodos do dia na estiagem. $\mathrm{Na}$ profundidade de $10 \mathrm{~cm}$, os valores médios de $\mathrm{O}_{2}$ foram maiores na enchente e menores na vazante, em razão de os solos estarem úmidos e a difusividade desses ficar prejudicada. $\mathrm{O}_{2}$ do solo tende a diminuir seus valores na cheia, quando o solo já está completamente alagado.

Um solo saturado não é uniformemente desprovido de oxigênio. A concentração de oxigênio, em geral, é alta na camada superficial oxidada (formada pela difusão de $\mathrm{O}_{2}$ pela atmosfera na água sobrejacente da inundação e, em seguida, no solo), onde ocorrem as reações de oxidação (Ponnomperumo, 1972; Moore \& Reddy, 1994). Processos de redução dominam abaixo da camada oxidada. Essa fina camada oxidada na superfície de solos alagados é importante para as transformações do $\mathrm{N}$, pois o $\mathrm{NH}_{4}{ }^{+}$pode oxidar-se para $\mathrm{NO}_{3}{ }^{-}$(nitrificação). O esgotamento do $\mathrm{NH}_{4}{ }^{+}$nessa camada causa sua difusão para cima (ascendente), em resposta à concentração do gradiente (Luo \& Zhou, 


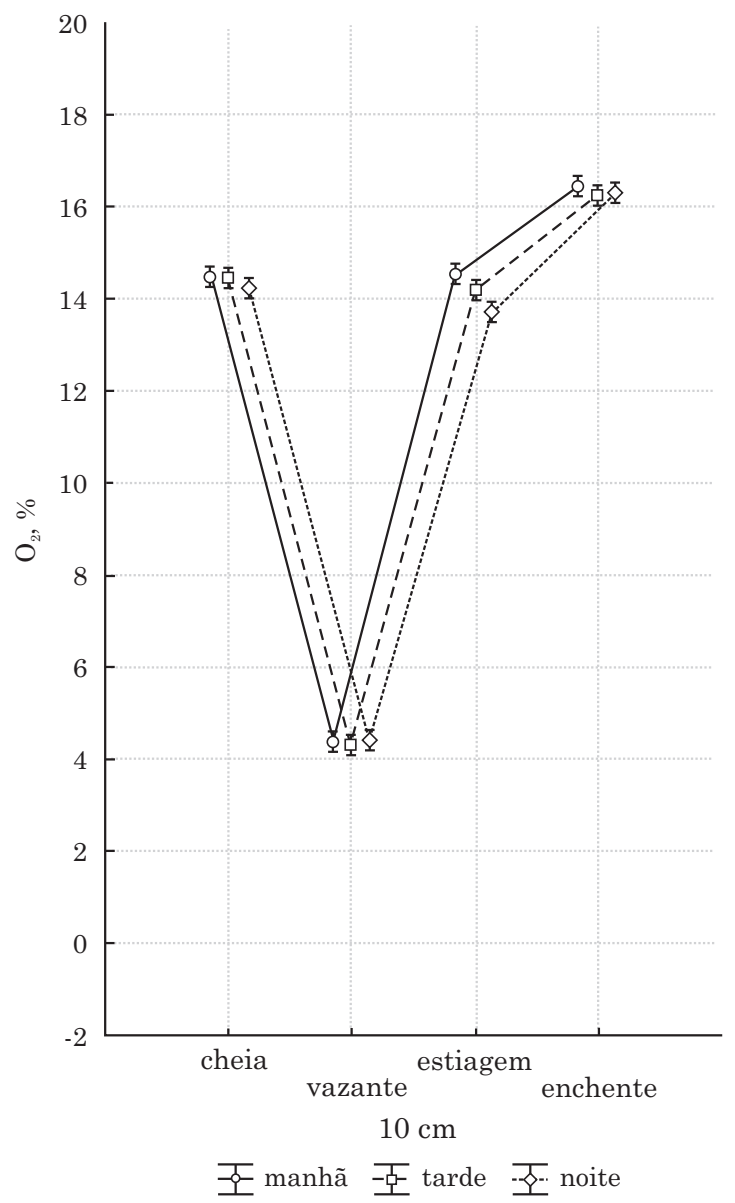

Figura 5. Médias nas estações vazante e cheia do $\mathrm{O}_{2}$, na profundidade de $10 \mathrm{~cm}$, num solo classificado como Planossolo Háplico alítico gleissólico, na fitofisionomia Cerrado sensu stricto. As barras expressam o intervalo de confiança a $95 \%$.

2006). Em razão da falha do sensor de $\mathrm{O}_{2}$ na profundidade $30 \mathrm{~cm}$, não foi possível comparar o oxigênio do solo nas duas profundidades, mas os dados de potencial redox (Figura 3) indicaram qual deve ser a tendência. Para a profundidade $10 \mathrm{~cm}$, os valores do potencial redox e do $\mathrm{O}_{2}$ indicaram que a enchente foi a estação mais oxidada.

Os valores da umidade do solo (Figura 6) diferiram ao longo do ciclo hidrológico nas duas profundidades estudadas. A umidade do solo diminuiu na estiagem, com menores valores médios, aumentando à medida que se aproximam as estações de chuva ( 15 e $25 \%$ nas estações de enchente e cheia, respectivamente), na profundidade de $10 \mathrm{~cm}$.

A umidade influencia a respiração do solo diretamente por meio de processos fisiológicos das raízes e dos microrganismos e indiretamente, via difusão dos substratos e $\mathrm{O}_{2}$ (Luo \& Zhou, 2006). A água nos poros do solo em altos níveis de umidade influencia a troca dos gases $\left(\mathrm{O}_{2}\right.$ e $\left.\mathrm{CO}_{2}\right)$, limitando a atividade microbiana. Na água, a taxa de difusão de

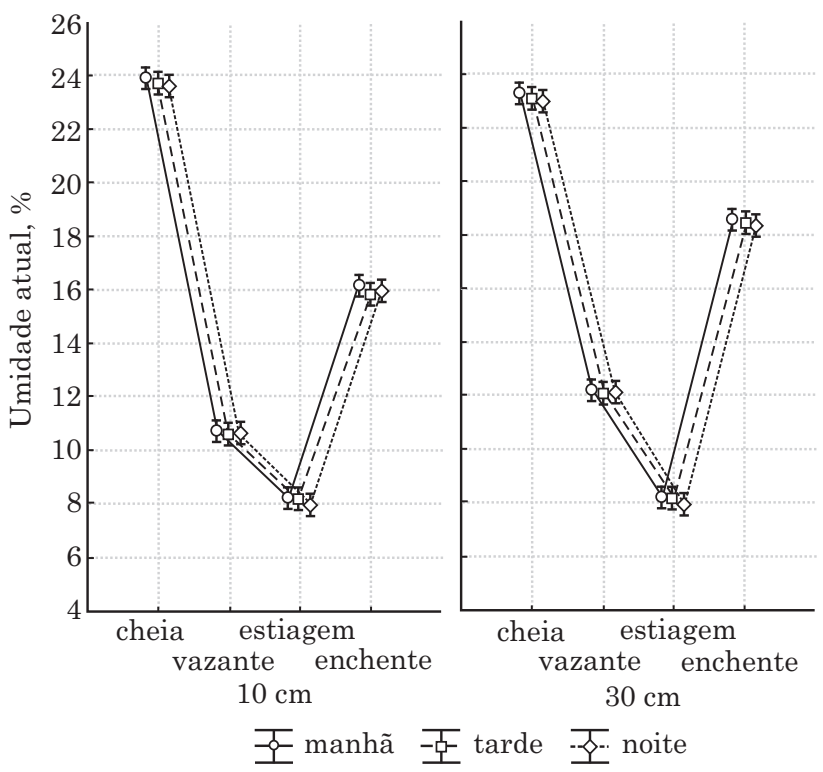

Figura 6. Médias, por ciclo hidrológico, da umidade do solo, nas profundidades de 10 e $30 \mathrm{~cm}$, num solo classificado como Planossolo Háplico alítico gleissólico, na fitofisionomia Cerrado sensu stricto. As barras expressam o intervalo de confiança a $95 \%$.

ambos os gases diminui em 10.000 vezes em relação à taxa de difusão no ar. Portanto, o movimento de difusão de ambos os gases diminui proporcionalmente à medida que os poros são ocupados por água (Luo \& Zhou, 2006).

Sendo o Pantanal uma planície sazonalmente inundável, existe um ciclo de umedecimento e secagem da planície especificamente na área de estudo. Em ambas as profundidades ocorrem a diminuição dos valores médios de umidade nas estações de vazante e estiagem, voltando a aumentar os valores médios nas duas estações subsequentes (enchente e cheia).

A variável potencial matricial solo (Figura 7) na profundidade de $10 \mathrm{~cm}$ foi estatisticamente diferente entre as estações, com menores valores nas estações de vazante e estiagem, -300 e $-325 \mathrm{kPa}$, respectivamente, e maiores valores nas de enchente $\mathrm{e}$ cheia, -150 e - $80 \mathrm{kPa}$, respectivamente. É importante ressaltar que em valores menores que $-600 \mathrm{kPa} o$ crescimento das raízes é reduzido. $O$ período da noite foi o que mais se diferenciou em todos os períodos ao longo do dia, nessa mesma profundidade (Figura 7).

$\mathrm{Na}$ profundidade de $30 \mathrm{~cm}$, não houve diferença estatística entre períodos do dia, mas houve diferenças entre as estações; na estiagem, a tensão do solo apresentou os menores valores $(-250 \mathrm{kPa})$. Observouse a mesma tendência do potencial matricial do solo com a umidade do solo (Figura 6), na profundidade de $30 \mathrm{~cm}$. O potencial matricial do solo na profundidade de $30 \mathrm{~cm}$ tende a diminuir à medida que a umidade do solo também reduz. 
A temperatura do solo (Figura 8 ) na região de estudo apresentou, para ambas as profundidades, os maiores valores médios na enchente e cheia $(27,3 \mathrm{e}$ $27,5^{\circ} \mathrm{C}$, na profundidade de $10 \mathrm{~cm}$, e 27,1 e $27,3^{\circ} \mathrm{C}$,

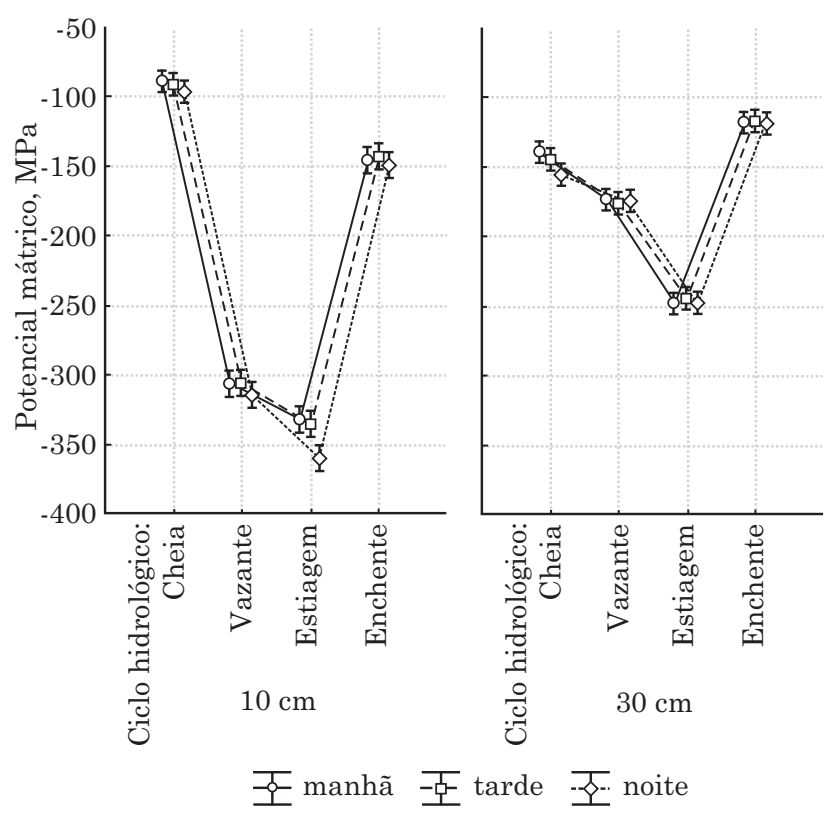

Figura 7 Médias do potencial matricial do solo, nas profundidades de 10 e $30 \mathrm{~cm}$, num solo classificado como Planossolo Háplico alítico gleissólico, na fitofisionomia Cerrado sensu stricto. As barras expressam o intervalo de confiança a $95 \%$.

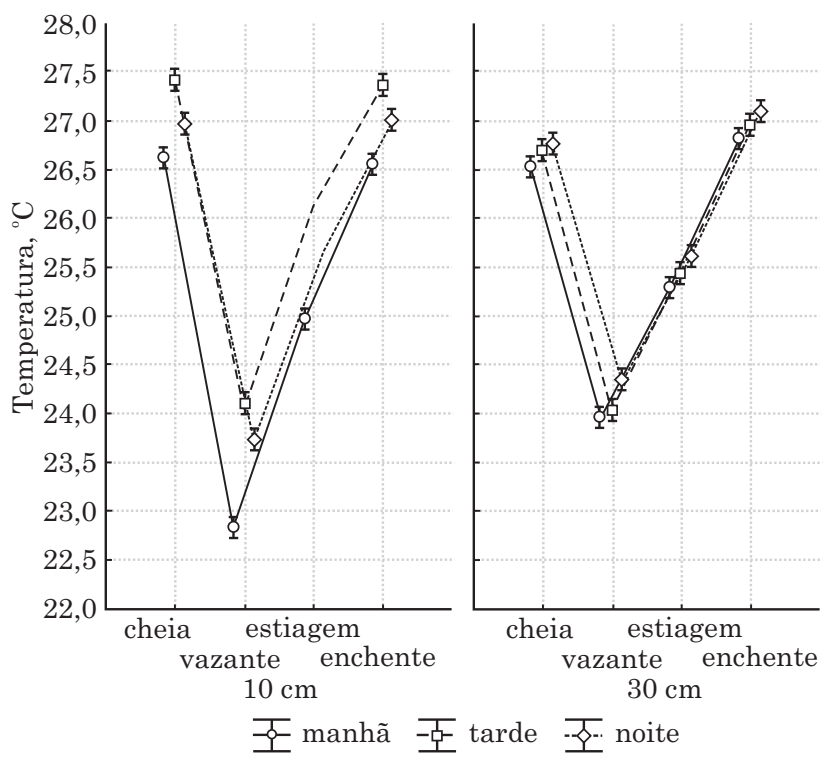

Figura 8. Médias, por estação do ciclo hidrológico, da temperatura do solo, nas profundidades de 10 e $30 \mathrm{~cm}$, num solo classificado como Planossolo Háplico alítico gleissólico, na fitofisionomia Cerrado sensu stricto. As barras expressam o intervalo de confiança a $95 \%$. na profundidade de $30 \mathrm{~cm}$ ). Houve diferença estatística entre as estações para ambas as profundidades. $\mathrm{Na}$ profundidade de $10 \mathrm{~cm}$, houve diferença estatística entre estações e entre os períodos da manhã, tarde e noite em todas as estações. Na profundidade de $30 \mathrm{~cm}$, os períodos da noite foram mais quentes que os outros períodos do dia. Isso foi estatisticamente diferente em quase todas as estações e resulta da transferência de calor da superfície do solo.

Nas regiões temperadas, evidencia-se forte relação entre temperatura do solo, conteúdo de água e concentrações de $\mathrm{CO}_{2}$ (Riveros-Iregui et al., 2007). Entretanto, os processos em solos tropicais são diferentes dos em solos das regiões temperadas, pois a temperatura varia pouco entre 10 e $30 \mathrm{~cm}$, apresentando claramente que a temperatura não é o fator mais importante na limitação dos processos biológicos.

\section{CONCLUSÕES}

1. Os resultados indicaram o potencial redox como ferramenta versátil para compreender as reações de oxirredução nos solos hidromórficos do Pantanal matogrossense, pois os valores diminuem quando o teor de umidade é elevado no solo, com redução da difusão do $\mathrm{O}_{2}$. Entretanto, nas estações mais secas do Pantanal, os valores positivos obtidos são típicos de solos aeróbicos, demonstrando predominarem condições não limitantes ao crescimento de plantas.

2. Os resultados evidenciaram que o estudo de variáveis complementares pode ser importante por permitir análise mais aprofundada das condições de hipersazonalidade, em que esses solos estão submetidos. $\mathrm{O}$ aumento da produção de $\mathrm{CO}_{2}$ no período da enchente e entre as diferentes profundidades; a diminuição dos valores de $\mathrm{O}_{2}$ quando o solo está alagado; e os menores valores de tensão do solo na estiagem são indicadores importantes para a compreensão dessas condições extremas. No caso específico da cordilheira estudada na RPPN SESC Pantanal, o potencial matricial e a umidade do solo foram as variáveis de maior importância para explicar a variação do conjunto de dados obtidos ao longo do ciclo hidrológico, o que indica que são determinantes nos processos biológicos desse solo tropical com hipersazonalidade hidríca.

\section{LITERATURA CITADA}

BEIRIGO, R.M.; VIDAL-TORRADO, P.; COUTO, E.G.; LUVEZUTI, C.B.; REIA, M.Y.; NASCIMENTO, V.O. \& PENTEADO, A.L.M. Processos redoximórficos e dinâmica de $\mathrm{P}$ em solos do Pantanal. In: CONGRESSO BRASILEIRO DE CIÊNCIA DO SOLO, 33., Solos nos biomas brasileiros: Sustentabilidade e mudanças climáticas, 33., Uberlândia, 2011. Anais... Uberlândia, SBCS, 2011. CD ROM 
BOWDEN, R.D.; NADELHOFFER, K.J.; BOONE, R.D.; MEILILLO, J.M. \& GARRISON, J.B. Contributions of aboveground litter, belowground litter, and root respiration to total soil respiration in a temperate mixed hardwood forest. Can. J. For. Res., 23:1402-1407, 1993.

CLARK, M.W.; McCONCHIE, D.; LEWIS, D.W. \& SAENGER, P. Redox stratification and heavy metal partitioning in Avicennia-dominated mangrove sediments: a geochemical model. Chem. Geol., 149:147-171, 1998.

CORDEIRO, J.L.P. Estrutura e heterogeneidade da paisagem de uma unidade de conservação no nordeste do Pantanal (RPPN SESC Pantanal), Mato Grosso, Brasil: Efeitos sobre a distribuição e densidade de antas (Tapirus terrestris) e de cervos-do-pantanal (Blastocerus dichotomus). Porto Alegre, Universidade Federal do Rio Grande do Sul, 2004. (Tese de Doutorado)

DE JONGE, H. \& MITTELMEIJER-HAZELEGER, M.C. Adsorption of $\mathrm{CO}_{2}$ and $\mathrm{N}_{2}$ on soil organic matter: nature of porosity, surface area, and diffusion mechanisms. Environ. Sci. Technol., 30:408-413, 1996.

GIRARD, P.; FANTIN-CRUZ, I.; DE OLIVEIRA, S.M.L. \& HAMILTON, S.K. Small-scale spatial variation of inundation dynamics in a floodplain of the Pantanal (Brazil). Hydrobiologia, 638:223-233, 2010.

HASENACK, H.; CORDEIRO, J.L.P. \& HOFMANN, G.S. O clima da RPPN SESC Pantanal, Relatório técnico. Porto Alegre, Instituto de Biociências, Centro de Ecologia, 2003. $31 \mathrm{p}$.

JANSEN, B.; NIEROP, K.G.J. \& VERSTRATEN, J.M. Mobilization of dissolved organic matter, aluminium and iron in podzol eluvial horizons as affected by formation of metal-organic complexes and interactions with solid soil material. Eur. J. Soil Sci., 55:287-297, 2004.

JASSAL, R.S.; BLACK, T.A.; DREWITT, G.B.; NOVAK, M.D.; GAUMONT-GUAY, D. \& NESIC, Z. A model of the production and transport of $\mathrm{CO}_{2}$ in soil: Predicting soil $\mathrm{CO}_{2}$ concentrations and $\mathrm{CO}_{2}$ efflux from a forest floor. Agric. For. Meteorol., 124:219-236, 2004.

JOHNSON, M.S.; BILLETT, M.F.; DINSMORE, K.J.; WALLIN, M.; DYSON, K.E. \& JASSAL, R.S. Direct and continuous measurement of dissolved carbon dioxide in freshwater aquatic systems-method and applications. Ecohydrology, 3:68-78, 2010.

JUNK, W.J.; BAYLEY, P.B. \& SPARKS, R.E. The flood pulse concept in river-floodplain systems. Canadian Special Public. Fish. Aquatic Sci., 106:110-127, 1989.

JUNK, W.J. \& CUNHA, C.N. Pantanal: A large South American wetland at a crossroads. Ecol. Eng., 24:391-401, 2005.

KALLESTAD, J.C.; MEXAL, J.G. \& SAMMIS, T.W. Comparison of galvanic and chemi-luminescent sensors for detecting soil air oxygen in flood-irrigated pecans. Soil Sci. Soc. Am. J., 72:758-766, 2008.

KELLER, J.K., WEISENHORN, P.B. \& MEGONIGAL, J.P. Humic acids as electron acceptors in wetland decomposition. Soil Biol. Biochem., 41:1518-1522, 2009.
LIESACK, W.; SCHNELL, S. \& REVSBECH, N.P. Microbiology of flooded rice paddies. FEMS Microbiol. Rev., 24:625$645,2000$.

LIMA, H.N.; MELLO, J.W.V.; SCHAEFER, C. \& KER, J.C. Dinâmica da mobilização de elementos em solos da Amazônia submetidos à inundação. Acta Amaz., 35:317-330, 2005.

LOBATO, L.A.O. Distribuição espacial de atributos pedológicos em áreas de cerrados mesotróficos no Pantanal de Poconé-MT. Cuiabá, Universidade Federal de Mato Grosso, 2000. 144p. (Dissertação de Mestrado)

LUO, Y. \& ZHOU, X. Soil respiration and the environment. San Diego, Academic Press, 2006. 328p.

MOORE, A. \& REDDY, K.R. Role of Eh and $\mathrm{pH}$ on phosphorus geochemistry in sediments of Lake Okeechobee, Florida. J. Environ. Qual., 23:955-964, 1994.

NEAMAN, A.; MOUÉLÉ, F.; TROLARD, F. \& BOURRIÉ, G. Improved methods for selective dissolution of Mn oxides: Applications for studying trace element associations. Appl. Geochem., 19:973-979, 2004.

CUNHA, C.N. \& JUNK, W.J. Year-to- year changes in water level drive the invasion of Vochysia divergens in Pantanal grasslands. Appl. Veg. Sci., 7:103-110, 2004.

PONNOMPERUMO, F.N. The chemistry of submerged soils. Adv. Agron., 24:29-96, 1972.

RANCOURT, D.G.; THIBAULT, P.-J.; MAVROCORDATOS, D. \& LAMARCHE, G. Hydrous ferric oxide precipitation in the presence of nonmetabolizing bacteria: Constraints on the mechanism of a biotic effect. Geochim. Cosmochim. Acta, 69:553-577, 2005.

RISK, D.; KELLMAN, L. \& BELTRAMI, H. A new method for in situ soil gas diffusivity measurement and applications in the monitoring of subsurface $\mathrm{CO}_{2}$ production. J. Geophys. Res., 113:G02018, 2008.

RIVEROS-IREGUI, D.A.; EMANUEL, R.E.; MUTH, D.J.; MCGLYNN, B.L.; EPSTEIN, H.E.; WELSCH, D.L.; PACIFIC, V.J. \& WRAITH, J.M. Diurnal hysteresis between soil $\mathrm{CO}_{2}$ and soil temperature is controlled by soil water content. Geophys. Res. Letters, 34:L17404, 2007.

SOUSA, R.O.; VAHL, L.C. \& OTERO, X.L. Química de solos alagados. In: MELLO, V.F. \& ALLEONI, L.R.F., eds. Química e mineralogia do solo. Parte II - Aplicações. Viçosa, MG, Sociedade Brasileira de Ciência do Solo, 2009. p.485-528.

VAISALA OYJ. User's guide vaisala CARBOCAP hand-held carbon dioxide meter GM70, Helsinki, Finland, Vaisala Oyj, Helsinki. 2008.

VEPRASKAS, M.J. \& FAULKNER, S.P. Redox chemistry of hydric soils. In: RICHARDSON, J.L. \& VEPRASKAS, M.J. Wetland soils: Genesis, hydrology, landscapes, and classification. Boca Raton, CRC Press, 2001. p.85-105.

VOLK, N.J. The effect of oxidation-reduction potential on plant growth. Agron. J., 31:665-670, 1939.

XU, L.; BALDOCCHI, D.D. \& TANG, J. How soil moisture, rain pulses, and growth alter the response of ecosystem respiration to temperature. Global Biogeochem. Cycles, 18:GB4002, 2004. 